\title{
Djamel Eddine Kouloughli (1947-2013)
}

Jean-Patrick Guillaume

\section{OpenEdition}

\section{Journals}

Édition électronique

URL : http://journals.openedition.org/beo/1255

DOI : 10.4000/beo.1255

ISSN : 2077-4079

\section{Éditeur}

Presses de l'Institut français du Proche-Orient

\section{Édition imprimée}

Date de publication : 30 avril 2014

Pagination : 17-22

ISBN : 978-2-35159-403-2

ISSN : 0253-1623

\section{Référence électronique}

Jean-Patrick Guillaume, « Djamel Eddine Kouloughli (1947-2013) », Bulletin d'études orientales [En ligne], LXII | 2014, mis en ligne le 26 mai 2014, consulté le 01 mai 2019. URL : http:// journals.openedition.org/beo/1255; DOI : 10.4000/beo.1255 


\section{DJAMEL EDDINE KOULOUGHLI (1947-2013)}

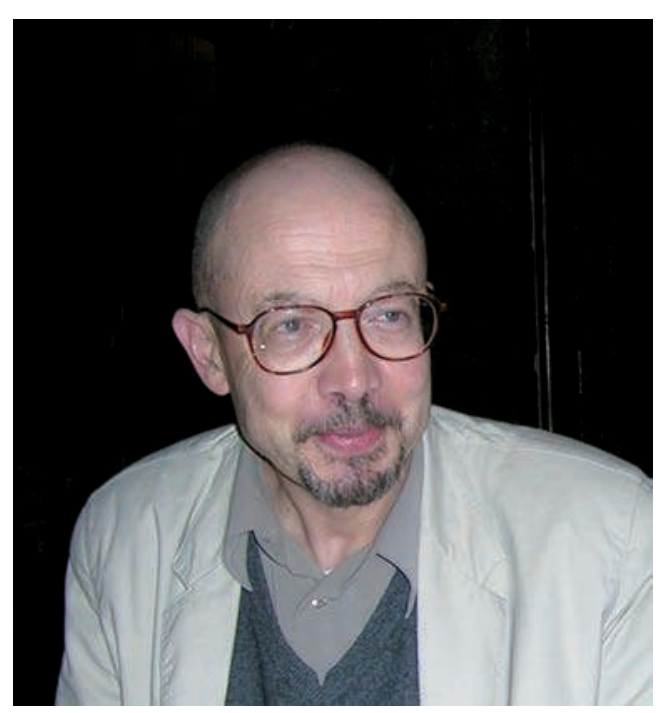

par Jean-Patrick GUILLAUME Professeur à l'Université Paris III

Né le 6 décembre 1947 à Constantine, Djamel Kouloughli avait commencé par suivre une formation d'angliciste, tout d'abord à l'Université d'Alger, puis, à partir de 1971, à l'Université Paris VII (Jussieu), avant de se réorienter vers la linguistique de l'arabe. Ses premiers travaux en ce domaine, et notamment sa thèse de Doctorat, portaient sur la morphologie et la phonologie, dans le cadre de la théorie générative, qui commençait alors à se diffuser dans les études arabes ; c'est de cette époque que date le début de sa collaboration scientifique - et de son amitié - avec Georges Bohas et moi-même. Parallèlement à ces travaux scientifiques, il est aussi conduit, pour financer ses études, à enseigner le français langue étrangère, puis l'arabe, dans le secteur associatif et privé ; il aimait à souligner 
combien l'expérience acquise dans ce domaine, ainsi que les connaissances en didactique des langues qu'il avait eu à cœur d'assimiler à cette occasion avaient été un élément important de sa formation.

Recruté au CNRS en 1982 dans la section "Langues et civilisations orientales », il est tout d'abord affecté à l'ERA 833, «Littérature contemporaine, linguistique et sémiologie arabes »; l'année suivante, il est reçu major de sa promotion à l'Agrégation d'arabe. Toutefois, les années 1980 voient une inflexion importante dans ses préoccupations de chercheur, qui le conduisent à prendre ses distances avec la morphophonologie et le modèle génératif. Cette évolution l'amène à obtenir, en 1986, son rattachement officiel au Laboratoire de linguistique formelle (UA 1026), dirigé alors par Antoine Culioli, unité avec laquelle il entretenait déjà des liens anciens et étroits, et où il avait fondé en 1984 le groupe Relpred (pour « Relations prédicatives »), rassemblant des linguistes et des informaticiens spécialistes du traitement automatique des langues, avec pour objectif d'élaborer des descriptions linguistiques formalisées, et notamment de modéliser les relations prédicatives dans diverses langues naturelles. C'est également de cette époque, dans l'environnement particulièrement vivant et dynamique de la tour centrale du campus de Jussieu, que datent ses premiers contacts avec le Laboratoire d'histoire des théories linguistiques (aujourd'hui UMR 7597), et en particulier avec Sylvain Auroux qui en était alors le directeur.

L'essor de la micro-informatique dans les années 1980 marque une étape cruciale dans le parcours de Djamel Kouloughli, qui a sans doute été l'un des premiers linguistes arabisants en France à prendre conscience des possibilités qu'offrait ce nouvel outil, mais aussi des conséquences théoriques et épistémologiques qu'impliquait sa mise en œuvre. Dans le cas de l'arabe, en particulier, la possibilité de constituer et d'exploiter de gros corpus textuels donnait enfin les moyens de refonder la description de la langue sur des données empiriques représentatives de l'usage réel de locuteurs réels, plutôt que sur les données et les exemples de la grammaire traditionnelle.

Encore fallait-il pour cela élaborer la méthodologie et les outils adéquats : il s'agissait pour lui d'une question cruciale, dans la mesure où les particularités phonographématiques de l'arabe ne permettaient pas de réutiliser tels quels, avec des résultats satisfaisants, les programmes conçus pour les langues à script latin. Il devait désormais consacrer à cette tâche une part importante de son activité scientifique ; les logiciels et les guides techniques qu'il a conçus dans ce cadre se caractérisent par le fait qu'ils s'appuient toujours sur une réflexion linguistique rigoureuse et exigeante, reflétant en creux son refus intransigeant des bricolages sans principe et des solutions ad hoc.

C'est toujours dans ce souci d'asseoir la description de l'arabe sur des bases empiriques solides et d'ouvrir des perspectives réelles d'élaboration d'une linguistique expérimentale qui le conduit à demander son rattachement au CEDEJ du Caire, où il séjourne de 1994 à 1996. Cette position privilégiée d'observateur sur le terrain est aussi pour lui l'occasion d'établir un important réseau de contacts, en Europe et dans le monde arabe, avec les chercheurs et les institutions intéressés à la constitution de gros corpus en arabe. 
À son retour en France, il crée le Centre d'étude des langues et littératures du monde arabe (CELLMA, UMR 8500), rattaché à l'ENS de Fontenay-St Cloud, qui vise à reconstituer un pôle pour la recherche arabisante dans la région Paris-Île de France. Toutefois, cette tentative, qui se heurte aux stratégies souvent très divergentes des individus et des institutions concernés, ne remplit que partiellement ses objectifs, en linguistique plus que dans les études littéraires. En outre, le déménagement de l'ENS à Lyon - alors que ses membres, notamment les enseignants-chercheurs, sont basés à Paris - complique un peu plus les choses et le CELLMA n'est pas renouvelé en 2001.

La carrière de Djamel Kouloughli se déroule alors au Laboratoire d'histoire des théories linguistiques, dont il est membre associé depuis les années 1980, et où nous assurons ensemble la responsabilité du projet « Tradition grammaticale arabe ». Toutefois, s'il consacre une part importante de ses activités à l'histoire et à l'épistémologie de la linguistique, il n'abandonne pas pour autant ses autres axes de recherche, qu'il s'agisse de la description de l'arabe, qu'il envisage dans toute la diversité de sa production écrite et dans la variété de ses formes historiques, ou de l'élaboration d'outils logiciels, notamment dans le domaine de la métrique arabe.

Sa disparition brutale, le 22 janvier 2013, a profondément bouleversé ses amis et ses partenaires scientifiques. L'étendue de ses connaissances et de ses compétences, la rigueur et la clarté de sa réflexion, mais aussi sa grande générosité intellectuelle et sa courtoisie raffinée faisaient de lui une grande figure des études arabes et de la linguistique.

\section{Bibliographie sélective}

\section{Ouvrages}

Contribution à la phonologie générative de l'arabe : le système verbal du parler arabe du Sra (Nord-Constantinois), thèse de $3^{\mathrm{e}}$ cycle, Université Paris VII, 1978.

The Arabic Linguistic Tradition, Londres, Routledge, 1990 [réimpr. Washington D. C., Georgetown University Press, 2006]. (avec G. Bohas et J.-P. Guillaume)

Lexique fondamental de l'arabe standard moderne, Paris, L'Harmattan, 1991.

Grammaire de l'arabe d'aujourd'hui, Paris, Presses-Pocket, 1994.

La philosophie du langage, Paris, PUF, 1996. (avec S. Auroux et J. Deschamps)

L'arabe, Paris, PUF, collection « Que sais-je », 2007.

Le résumé de la grammaire arabe par Zamakš̌arī, Lyon, ENS éditions, 2007.

\section{Articles et contribution à des ouvrages collectifs}

«Sur le traitement des glides dans la phonologie de l'arabe standard », Analyses/Théorie 1979/1, p. 54-93.

«Processus accentuels en arabe (parlers du Caire, de Damas et arabe classique) » (avec Georges Bohas), Analyses/théorie, 1981/1, p.1-59. 
«Sur le système vocalique du damascène », Analyses/théorie 1981/3, p.36-46. (avec Georges Bohas)

"Sur la phonographématique arabe », Analyses/théorie, 1982/1, p. 79-151.

«À propos de lafž et mainā», Bulletin d'Études Orientales 35 (1985), p. 43-63.

«Sur la structure interne des syllabes ‘lourdes' en arabe classique », Revue Québécoise de Linguistique 16-1 (1986), p. 129-154.

"Les particules ont-elles un sens? Autour d'une controverse dans la tradition grammaticale arabe ", Bulletin de Linguistique appliquée et générale 13 (1987), p. 215-237.

"Vers un traitement automatique de la prédication verbale en arabe standard moderne ", Revue internationale du traitement automatique du langage 28-1 (1987), p. 23-45.

«Renouvellement énonciatif et valeur aoristique : à propos de l'opposition $m \bar{a}$ / lam en arabe », Langues de l'Orient ancien 1 (1988), p. 49-72.

«L'analyse linguistique dans la tradition arabe », in S. Auroux (éd.), Histoire des idées linguistiques, vol. 1, Paris, Mardaga, 1990, p. 260-282. (avec G. Bohas et J.-P. Guillaume)

« La thématique du langage dans la Bible », in S. Auroux (éd.), Histoire des idées linguistiques, vol. 1, Paris, Mardaga, 1990, p. 65-78.

«Les débuts de la grammaire hébraïque », in S. Auroux (éd.), Histoire des idées linguistiques, vol. 1, Paris, Mardaga, 1990, p. 283-292. (avec G. Bohas et J.-P. Guillaume)

«Why is there no 'true' philosophy of linguistics? », Language and Communication II/3 (1991), p. 151-163. (avec S. Auroux)

«Indéfini et structure thématique en arabe », Faits de langue 4 (1994), p. 169-175.

«Sur la représentation des relations syntaxiques en traitement automatique des langues naturelles » in J. Bouscaren, J.-J. Franckel et S. Robert Langues et langage : problèmes et raisonnements en linguistique. Mélanges offerts à A. Culioli, Paris, PUF, 1995.

«La phrase dans la tradition grammaticale arabe », Mémoires de la Société de linguistique de Paris, nouvelle série, t. III (1995), p. 81-91.

«Contre la synonymie : présentation et traduction française annotée du premier chapitre de l'ouvrage d'Abū Hilāl al-'Askarī (m. vers 1005) Kitāb al-Furūq fïl-luġa », Histoire Epistémologie Langage 19/2 (1997), p. 155-176.

«Une théorie "opérationnaliste" des cas morphologiques de l'arabe est-elle possible ?», Histoire Épistémologie Langage 20/2 (1998), p. 35-42.

« Y a-t-il une syntaxe dans la Tradition Arabe? », Histoire Épistémologie Langage 21/2 (1999), p. 45-64.

«Sur l'analyse syllabique automatique de l'arabe », Langues et littératures du monde arabe 1/1 (2000), p. 2942.

«Le modèle d'analyse de l'énoncé des rhétoriciens arabes dans le 'ilm al-ma‘ān̄̄ », Histoire Épistémologie Langage 22/2 (2000), p. 97-104.

«Of Linguists and Doorkeepers : a short response to Mr. Owens », Histoire Épistémologie Langage 22/2 (2000), p. 127-132.

«Sur le statut linguistique du tanwīn, contribution à l'étude du système déterminatif de l'arabe », Arabica 48 (2001), p. 20-50. 
«Réflexions sur l'origine du terme ğumla dans la tradition grammaticale arabe », in B. Colombat et M. Savelli (éd.), Actes du colloque «Métalangage et théories grammaticales», Peeters, Louvain, 2001, p. 577-589.

« Towards a systematic corpus analysis of Arabic poetry », in C. Michaux et M. Dominicy (éd.), Linguistic approaches to poetry, Belgian Journal of Linguistics 15 (2001), p. 103-112. (avec G. Bohas)

"L'influence mu'tazilite sur la naissance et le développement de la rhétorique arabe », Arabic Science and Philosophy 12 (2002), p. 217-239.

«On locative sentences in Arabic », Zeitschrift für Arabische Linguistik 41 (2002), p. 7-26.

«Le texte arabe du Roman de Baybars : premier survol du corpus électronique », Arabica 51/1 (2004), p. 121-143.

«Autour du préambule du Kitāb de Sībawayhi », Langues et littératures du monde arabe 5 (2005), p. 9-16. En ligne : http://w3.ens-lsh.fr/llma/sommaires/LLMA5_Autour_du_preambule.pdf

« Préambule du Kitāb de Sībawayhi : traduction française », Langues et littératures du monde arabe 5 (2005), p. 9-16. http://w3.ens-lsh.fr/llma/sommaires/LLMA5_04_Preambule.pdf

«Introduction pratique à la constitution et à l'exploitation de corpus électroniques en langue arabe ( $1^{\text {re }}$ partie) », Langues et littératures du monde arabe 5 (2005), p. 231-287. En ligne : http://w3.ens-lsh.fr/ llma/sommaires/LLMA5_12_\%20D-E_Kouloughli.pdf

« Inflexion », in K. Versteegh et al. (éd.), Encyclopedia of Arabic Language and Linguistics, vol. 2, Leiden, Brill, 2006.

« Lafz (phonetic form)», in K. Versteegh et al. (éd.), Encyclopedia of Arabic Language and Linguistics, vol. 2, Leiden, Brill, 2006.

« Introduction pratique à la constitution et à l'exploitation de corpus électroniques en langue arabe ( $2^{e}$ partie) », Langues et littératures du monde arabe 6 (2007), p. 97-114. En ligne : http://w3.ens-lsh.fr/ llma/sommaires/LLMA6_art_Kouloughli.pdf

«Ma'nā (Meaning)», in K. Versteegh et al. (éd.), Encyclopedia of Arabic Language and Linguistics, vol. 3, Leiden, Brill, 2007.

«Sur la valeur du tanwīn : nouvelle contribution à l'étude du système déterminatif en arabe », Arabica 54 (2007), p. 94-131.

"Moyen arabe et questions connexes », La clé des langues, publié le 23 novembre 2007, mis à jour le 13 septembre 2009, http://cle.ens-lyon.fr/arabe/moyen-arabe-et-questions-connexes-30683.kjsp?RH=CDL_ARA120000

« Ernest Renan, un antisémitisme savant », Histoire Épistémologie Langage 29/2 (2007) p. 91-112.

«Introduction pratique à la constitution et à l'exploitation de corpus électroniques en langue arabe ( $3^{\mathrm{e}}$ partie) », Langues et littératures du monde arabe 7 (2008), p. 75-93. En ligne : http://w3.ens-lsh.fr/llma/ sommaires/LLMA_7_05_Kouloughli.pdf

«Introduction pratique à la constitution et à l'exploitation de corpus électroniques en langue arabe (4e partie) », Langues et littératures du monde arabe 8 (2009), p. 117-133. En ligne : http://w3.ens-lsh.fr/ llma/sommaires/LLMA_8_11_Kouloughli_Corpus.pdf

«Langues sémitiques et traduction : critique de quelques vieux mythes », in I. Rosier-Catach et al. (éd.) Les Grecs, les Arabes et nous : enquête sur l'islamophobie savante, Paris, Fayard, 2009, p. 79-118. 
"Traitement automatique de la métrique arabe :état des lieux et perspectives », Bulletin d'études orientales 59 (2010) (D. Choukr \& B. Paoli (éd.), Métrique arabe : état des lieux et perspectives, actes de la table ronde internationale, Damas, Institut français du Proche-Orient, 27 et 28 avril 2007), p. 17-32.

«Le Coran : quelques données lexico-statistiques », Langues et littératures du monde arabe 8 (2009), p. 61-73. En ligne : http://w3.ens-lsh.fr/llma/sommaires/LLMA_8_06_Kouloughli_Coran.pdf (avec G. Bohas).

«Vers un corpus électronique du Kitāb de Sībawayhi », Langues et littératures du monde arabe 9 (2011), p. 60-67. En ligne : http://w3.ens-lsh.fr/llma/sommaires/LLMA9_06_Ko_Bo\%20DEF.pdf

«Segmentation and Tagging of Vocalized Arabic Texts », Langues et littératures du monde arabe 9 (2011), p. 68-83. En ligne : http://w3.ens-lsh.fr/llma/sommaires/LLMA9_07_Kouloughli\%20DEF.pdf

« De la couleur en arabe », Cahiers du Léopard d'or 13 (2013), p. 131-181.

"Essai de lecture kuhnienne de l'histoire de la tradition grammaticale arabe ", à paraitre in S. Archaimbault, J. M. Fournier et V. Raby (éd.), Penser l'histoire des savoirs linguistiques. Hommage à Sylvain Auroux, Lyon, ENS-Éditions. 